\title{
Transverse Momentum Distributions of Quarks from the Lattice using Extended Gauge Links
}

\author{
Bernhard U. Musch ${ }^{* a}$, Philipp Hägler ${ }^{a}$, Andreas Schäfer ${ }^{b}$, Meinulf Göckeler ${ }^{b}$, \\ Dru B. Renner ${ }^{c}$, John W. Negele ${ }^{d}$, LHPC (Lattice Hadron Physics Collaboration) \\ a Institut für Theoretische Physik T39, Physik-Department, Technische Universität München, \\ James-Franck-Straße, D-85747 Garching, Germany \\ ${ }^{b}$ Institut für Theoretische Physik, Universität Regensburg, \\ D-93040 Regensburg, Germany \\ ${ }^{c}$ Department of Physics, University of Arizona, \\ 1118 E 4th Street, Tucson, AZ 85721, USA \\ ${ }^{d}$ Center for Theoretical Physics, Massachusetts Institute of Technology, \\ Cambridge, MA02139, USA \\ E-mail: bmusch@ph.tum. de
}

\begin{abstract}
We present preliminary numerical studies in Lattice QCD related to the intrinsic transverse momentum distribution of partons in the nucleon. We employ non-local operators, consisting of spatially separated quark creation and annihilation operators connected by a straight Wilson line. A clear signal is already obtained from a small number of configurations at a pion mass $m_{\pi} \approx 600$ $\mathrm{MeV}$. As an example, we demonstrate that we can obtain the first $x$-moment of the transverse momentum dependent parton distribution function $f_{1}^{n=1}\left(\vec{k}_{T}\right)$ from our data. Our results, which are not renormalized, show a Gaussian-like distribution. The root mean squared transverse momentum is $\sqrt{\left\langle\vec{k}_{T}^{2}\right\rangle} \approx 560 \mathrm{MeV}$ for a Gaussian fit, close to phenomenological values.
\end{abstract}

The XXV International Symposium on Lattice Field Theory

July 30 - August 42007

Regensburg, Germany

${ }^{*}$ Speaker. 


\section{Introduction}

The Bjorken- $x$-dependence of parton distribution functions (PDFs) has been investigated by several lattice collaborations in recent years. In these studies, the lattice operators probing the nucleon always represent a local continuum operator. Spatial separations between the quark annihilation and creation operator on the lattice only appear in the context of discretized covariant derivatives.

In this study, we explore the possibility to examine the dependence on the intrinsic parton momentum $\vec{k}_{T}$ as well. For the purpose of calculating transverse momentum dependent parton distribution functions (TMDPDFs), we investigate non-local operators, constructed from quark and antiquark fields which are spatially separated. Gauge invariance is ensured by introduction of a straight gauge link (Wilson line) connecting the quark fields.

\section{TMDPDFs in SIDIS experiments}

In experimental processes like semi-inclusive deep inelastic scattering (SIDIS), one factorizes dominant diagrams into hard, perturbative processes and soft, non-perturbative parts $[1,2,3]$, represented as shaded areas in fig. 1 a). The lower soft part can be parameterized in terms of nucleon TMDPDFs and corresponds to a correlator

$$
\begin{aligned}
& \Phi^{[\Gamma]}(k, P, S) \equiv \int \frac{d^{4} \ell}{2(2 \pi)^{4}} e^{i k \cdot \ell}\left\langle P, S\left|\mathscr{O}^{\Gamma}(\ell)\right| P, S\right\rangle, \\
& \text { where } \quad \begin{aligned}
\mathscr{O}^{\Gamma}(\ell) & \equiv \bar{q}(0) \Gamma \mathscr{U}_{[0, \ell]} q(\ell)
\end{aligned}
\end{aligned}
$$

Here $|P, S\rangle$ represents a nucleon state of momentum $P$ and spin $S, \Gamma$ is a Dirac matrix and $k$ is the quark momentum. $\mathscr{U}_{[0, \ell]}$ is a gauge link connecting the quark operators. For SIDIS, the gauge link runs to infinity and back, as illustrated in fig. 1 b), see, e.g., ref. [4].

a)

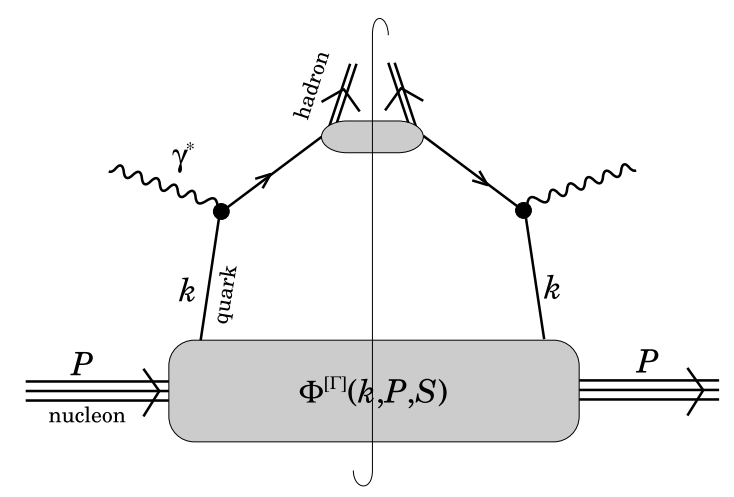

b)

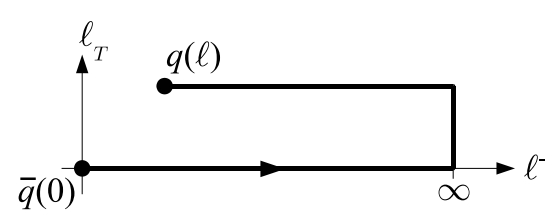

c)

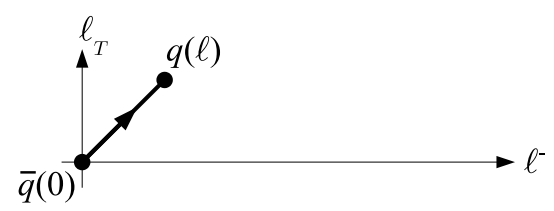

Figure 1: a) Factorized tree level diagram of semi-inclusive deep inelastic scattering (SIDIS), b) Gauge link to infinity and back as in SIDIS, c) Straight gauge link 


\section{First approach to TMDPDFs from the lattice}

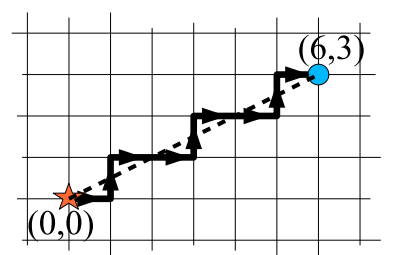

Figure 2: Step-like link path for $\vec{l}=(6,3,0)$.

The idea of this study is to extract TMDPDFs from matrix elements $\left\langle P, S\left|\mathscr{O}^{\Gamma}(\ell)\right| P, S\right\rangle$ calculated directly on the lattice. So far we restrict ourselves to straight gauge link operators: in the continuum limit, the gauge link follows a straight path connecting quark annihilation and creation operators, see fig. $1 \mathrm{c}$ ). The matrix elements thus calculated do not correspond exactly to those relevant for SIDIS, but the resulting TMDPDFs have an interesting probabilistic interpretation [5]. On the lattice, the gauge link $\mathscr{U}_{[0, \ell]}$ in the operator $\mathscr{O}_{\text {lat }}^{\Gamma}(\ell)$ is a product of link variables. Since we work in Euclidean space-time, we can only evaluate spatial quark separations $\ell=(0, \vec{\ell})$. Therefore we restrict our lattice operators to a single slice $\tau$ in Euclidean time. For quark separation $\vec{l}$ which do not lie on the $x, y$ or $z$ axis, we can approximate a straight connection with a step-like link path, see fig. 2 (inset).

To extract a signal for nucleon matrix elements of the operators in eq. (2.2), we form ratios of nucleon three-point and two-point functions

$$
R_{\Gamma}(\tau ; \vec{P}, \vec{\ell})=\frac{\Gamma_{\alpha \beta}^{3 \mathrm{pt}}\left\langle B_{\beta}\left(t_{\mathrm{sink}}, \vec{P}\right) \mathscr{O}_{\mathrm{lat}}^{\Gamma}(\vec{\ell} ; \tau) \bar{B}_{\alpha}(0, \vec{P})\right\rangle}{\Gamma_{\alpha \beta}^{2 \mathrm{pt}}\left\langle B_{\beta}(t, \vec{P}) \bar{B}_{\alpha}(0, \vec{P})\right\rangle},
$$

where $\Gamma^{2 \mathrm{pt}}$ and $\Gamma^{3 \mathrm{pt}}$ are suitable nucleon spin projection matrices ${ }^{1}$, and where $B(t, \vec{P})$ is a nucleon interpolating operator, composed of $u$ and $d$ quark operators. Nucleon source and sink are placed at

a)

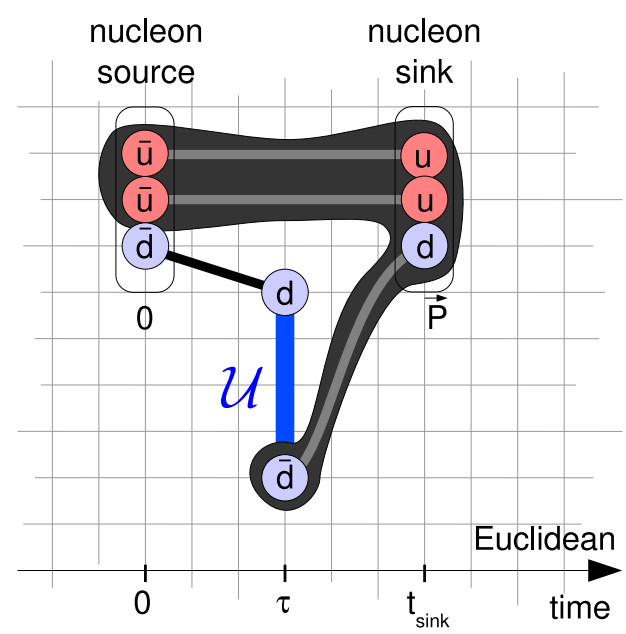

b)

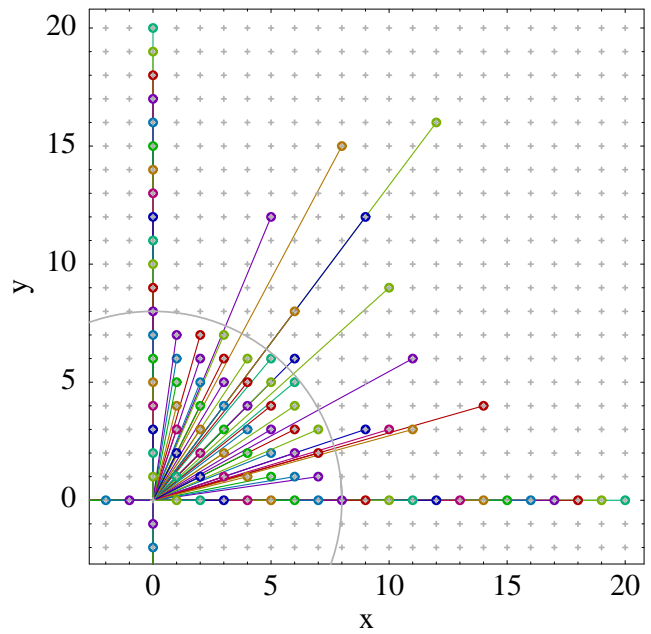

Figure 3: a) Evaluation of the three-point function in the numerator of eq. (3.1) on the lattice (schematic), here for an operator $\mathscr{O}_{\text {lat }}^{\Gamma}$ with d-quarks. Only one of the two possible connected contractions of quark fields is shown. All-to-all propagators are avoided by combining three of the quark propagators into a sequential propagator (dark area). b) Overview of quark separations $\vec{\ell}$ in the $\mathrm{x}$-y-plane used in this investigation. We have calculated all quark separations which lie on the $\mathrm{x}$ - or $\mathrm{y}$-axis, up to a length of 20 lattice units. In the first quadrant, we have included all quark separations up to a length of 8 lattice units (inner grey circle) and a selection of longer ones.

\footnotetext{
${ }^{1} \mathrm{LHPC}$ uses $\Gamma^{2 \mathrm{pt}}=\frac{1}{2}\left(\mathbb{1}+\gamma_{4}\right)$ and $\Gamma^{3 \mathrm{pt}}=\frac{1}{2}\left(\mathbb{1}+\gamma_{4}\right)\left(\mathbb{1}+i \gamma_{5} \gamma_{3}\right)$
} 
times $t_{\text {source }}=0$ and $t_{\text {sink }}$, respectively. The transfer matrix formalism reveals that at a sufficiently large distance from source and sink $\left(0 \ll \tau \ll t_{\text {sink }}\right)$, the ratio $R_{\Gamma}(\tau ; \vec{P}, \vec{\ell})$ becomes $\tau$-independent. This plateau value is directly related to the value of the matrix element $\left\langle P, S\left|\mathscr{O}_{\text {lat }}^{\Gamma}(\ell)\right| P, S\right\rangle$.

For the evaluation of eq. (3.1), we apply the standard technique as described, e.g., in ref. [6], based on products of propagators and sequential propagators as illustrated in fig. $3 \mathrm{a}$ ). In the following, we present results for isovector operators $(q=u-d)$, where disconnected contributions are absent.

\section{Test setup}

In our first numerical test calculations, we use 84 MILC gauge configurations from the NERSC archive $[8,9]$. The configurations were produced with the AsqTad improved staggered quark action with $2+1$ flavors. The lattice dimensions are $L^{3} \times T=20^{3} \times 64$, with a lattice spacing $a \approx 0.124 \mathrm{fm}$. The quark masses are $a m_{u, d}=0.030$, and $a m_{s}=0.050$. The gauge configurations have been HYP smeared and bisected in the temporal direction, and we have selected only the time slices $0 \ldots 31$. We are using unsmeared propagators and sink-smeared sequential propagators previously calculated by the LHPC group for these chopped gauge configurations (see, e.g. [7]). The propagators have been calculated using domain wall fermions, with the quark mass tuned such that the pion mass $m_{\pi} \approx 596 \mathrm{MeV}$ is approximately equal to the Goldstone pion mass for the staggered sea quark action. The source-sink separation is $t_{\text {sink }}-t_{\text {source }}=10$. LHPC has calculated sequential propagators for two nucleon momenta $\vec{P}=(0,0,0)$ and $\vec{P}=(-1,0,0)$. The latter corresponds to a momentum of $500 \mathrm{MeV}$ in physical units.

We have explored a number of link paths in all directions. Fig. 3 b) illustrates our selection of quark separations $\vec{\ell}$ in the $x$-y-plane. For our test runs, we have chosen two Dirac structures in the operator: the vector case $\Gamma=\gamma_{4}$ and the axial vector case $\Gamma=\gamma_{3} \gamma_{5}$.

a)

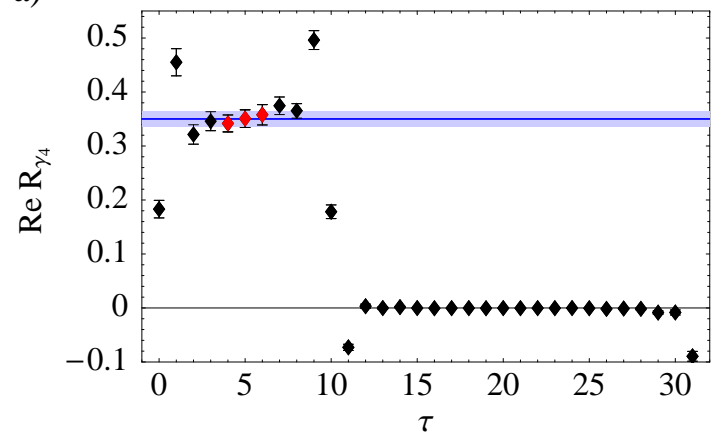

b)

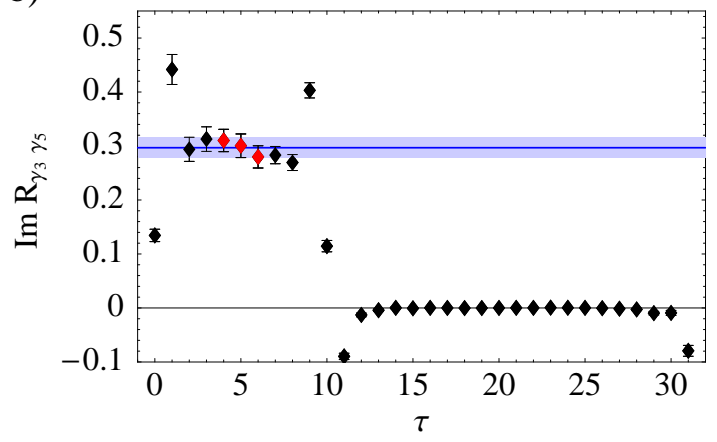

Figure 4: Sample plateau plots: $R_{\Gamma}(\tau ; \vec{P}, \vec{\ell})$ is plotted versus $\tau$. The horizontal line and the error band indicate the plateau value and its error, extracted from the points (marked red), at $\tau=4,5$ and 6 . a) Real part of $R_{\Gamma}(\tau ; \vec{P}, \vec{\ell})$ for $\Gamma=\gamma_{4}$, nucleon momentum $\vec{P}=(0,0,0)$, and a link path five units long in $x$ direction, i.e. $|\vec{\ell}|=5$. b) Imaginary part of $R_{\Gamma}(\tau, \vec{P}, \vec{\ell})$ for $\Gamma=\gamma_{3} \gamma_{5}$, nucleon momentum $\vec{P}=(0,0,0)$, and the link path shown in fig. 2 , i.e. $|\vec{\ell}|=6.7$. 


\section{Preliminary Results}

In fig. 4 we show two sample plots of the ratio $R_{\Gamma}(\tau ; \vec{P}, \vec{\ell})$ versus $\tau$. We obtain clean plateaus. In order to extract the plateau value $R_{\Gamma}(\vec{P}, \vec{\ell})$, we average over time slices at $\tau=4,5$ and 6 .

Fig. 5 a) shows all results $R_{\Gamma}(\vec{P}, \vec{\ell})$ for $\Gamma=\gamma_{4}$ and $\vec{P}=(0,0,0)$ for the 263 evaluated link paths. The signal is quite good, even for longer quark separations. We find that the correlator primarily depends on the separation $|\vec{\ell}|$ between quark annihilation and creation operator.

In figures $5 \mathrm{~b}$ ) (vector case, unpolarized) and 6 a) (axial vector case, polarized) we select only link paths lying in the $x-y$-plane. Furthermore, we identify groups of link paths which transform into one another under rotation or reflection in the $x-y$-plane, and take the group average. In fig. 6 b) we show an example at non-zero nucleon momentum. For quark separations $\vec{\ell}$ on the positive

a)



b)

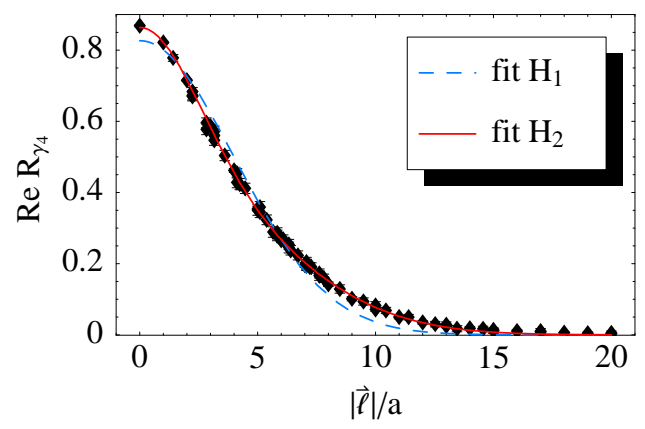

Figure 5: a) Results for $\Gamma=\gamma_{4}, \vec{P}=(0,0,0)$. We plot $\operatorname{Re} R_{\gamma_{4}}(\vec{P}, \vec{\ell})$ for all link paths versus the separation $|\vec{\ell}|$ of quark creation and annihilation operator. b) $\operatorname{Re} R_{\gamma_{4}}(\vec{P}=0, \vec{\ell})$ versus $|\vec{\ell}|$ for link paths in the x-y-plane. Results for link paths which transform into one another under rotation or reflection have been averaged. Dashed turquoise curve: fit to the data with a single Gaussian function $H_{1}(|\vec{\ell}|)$, see eq. (5.1). Solid red curve: fit with the superposition of two Gaussian functions $H_{2}(|\vec{\ell}|)$. The parameters determined from the fits are listed in tables 1 and 2.

a)

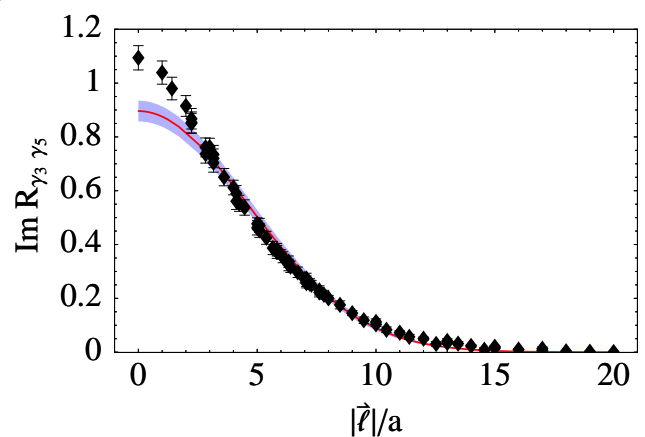

b)

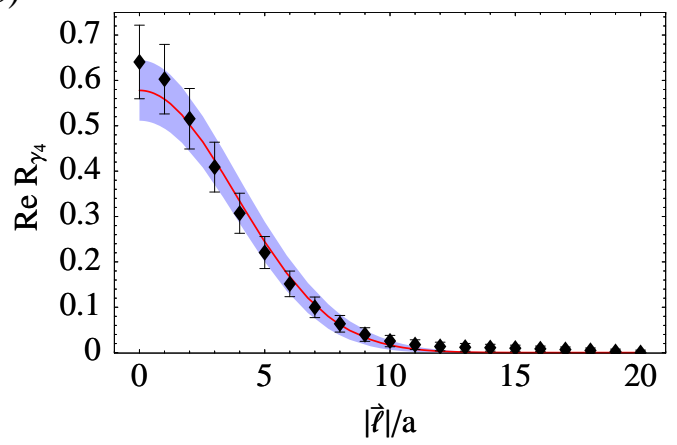

Figure 6: a) $\operatorname{Im} R_{\Gamma}$ versus $|\vec{\ell}|$ for $\Gamma=\gamma_{3} \gamma_{5}$ (axial vector), momentum $\vec{P}=(0,0,0)$ and link paths in the $\mathrm{x}$-y-plane. Results for link paths which transform into one another under rotation or reflection have been averaged. b) $\frac{1}{2} \operatorname{Re}\left\{R_{\Gamma}(\vec{\ell})+R_{\Gamma}(-\vec{\ell})\right\}$ for $\Gamma=\gamma_{4}, \vec{\ell}$ on the positive $x$-axis, and non-zero nucleon momentum $\vec{P}=(-1,0,0)$. In both plots, the data have been fitted with a single Gaussian function $H_{1}(|\vec{\ell}|)$, see eq. (5.1). Parameters determined from the fits are listed in table 1. 
$x$-axis, nucleon momentum $\vec{P}=(-1,0,0)$ and $\Gamma=\gamma_{4}$, we plot $\frac{1}{2} \operatorname{Re}\left\{R_{\Gamma}(\vec{\ell})+R_{\Gamma}(-\vec{\ell})\right\}$. Here we refrain from averaging over the whole $x-y$-plane, because the parameterization indicates that the value is not invariant with respect to the link direction.

We have tested the following fit functions to parameterize the $|\vec{\ell}|$ dependence:

$$
H_{1}(|\vec{\ell}|):=C_{1} \exp \left(-\frac{|\vec{\ell}|^{2}}{\sigma_{1}^{2}}\right), \quad H_{2}(|\vec{\ell}|):=C_{1} \exp \left(-\frac{|\vec{\ell}|^{2}}{\sigma_{1}^{2}}\right)+C_{2} \exp \left(-\frac{|\vec{\ell}|^{2}}{\sigma_{2}^{2}}\right) .
$$

The resulting fit parameters are listed in tables 1 and 2. We observe that the data can be well described by the sum of Gaussians in $\mathrm{H}_{2}$.

\section{A first glimpse of TMDPDFs from the lattice}

We can use the parameters of the fits in fig. $5 \mathrm{~b})$ to calculate the first $x$-moment $(n=1)$ of the intrinsic transverse momentum dependence of the parton density $f_{1}$ :

$$
f_{1}^{n=1}\left(\vec{k}_{T}\right) \equiv \int_{-1}^{1} d x \int_{-\infty}^{\infty} d k^{-}\left(\int \frac{d^{4} \ell}{2(2 \pi)^{4}} e^{i k \cdot \ell}\left\langle P, S\left|\bar{q}(0) \gamma^{+} \mathscr{U}_{[0, \ell]} q(\ell)\right| P, S\right\rangle\right) .
$$

It turns out that

$$
f_{1, \text { lat }}^{n=1}\left(\vec{k}_{T}\right)=\int \frac{d^{2} \vec{\ell}_{T}}{(2 \pi)^{2}} e^{-i \vec{k}_{T} \cdot \vec{\ell}_{T}} R_{\gamma_{4}}\left(\vec{P}=0,\left|\vec{\ell}_{T}\right|\right)
$$

\begin{tabular}{l||c|c||c|c|c} 
fig. & $\Gamma$ & $\vec{P}$ & $C$ & $\sigma$ & $2 / \sigma$ \\
\hline 5b) dashed & $\gamma_{4}$ & $(0,0,0)$ & $0.826 \pm 0.005$ & $(5.64 \pm 0.12) a=0.70 \mathrm{fm}$ & $(563 \pm 12) \mathrm{MeV}$ \\
6 a) & $\gamma_{3} \gamma_{5}$ & $(0,0,0)$ & $0.90 \pm 0.04$ & $(6.58 \pm 0.12) a=0.82 \mathrm{fm}$ & $(484 \pm 9) \mathrm{MeV}$ \\
6b) & $\gamma_{4}$ & $(-1,0,0)$ & $0.58 \pm 0.07$ & $(5.4 \pm 0.5) a=0.67 \mathrm{fm}$ & $(589 \pm 46) \mathrm{MeV}$
\end{tabular}

Table 1: Fit parameters determined from the single Gaussian fits with $H_{1}$ shown in fig. 5 b) and 6 .

\begin{tabular}{l||c|c||c|c|c||c|c|c} 
fig. & $\Gamma$ & $\vec{P}$ & $C_{1}$ & $\sigma_{1}$ & $2 / \sigma_{1}$ & $C_{2}$ & $\sigma_{2}$ & $2 / \sigma_{2}$ \\
\hline 5b) solid & $\gamma_{4}$ & $(0,0,0)$ & 0.49 & $7.3 a$ & $(433 \pm 15) \mathrm{MeV}$ & 0.37 & $3.4 a$ & $(945 \pm 41) \mathrm{MeV}$
\end{tabular}

Table 2: Fit parameters determined from the double Gaussian fit with $H_{2}$ shown in fig. 5 b)

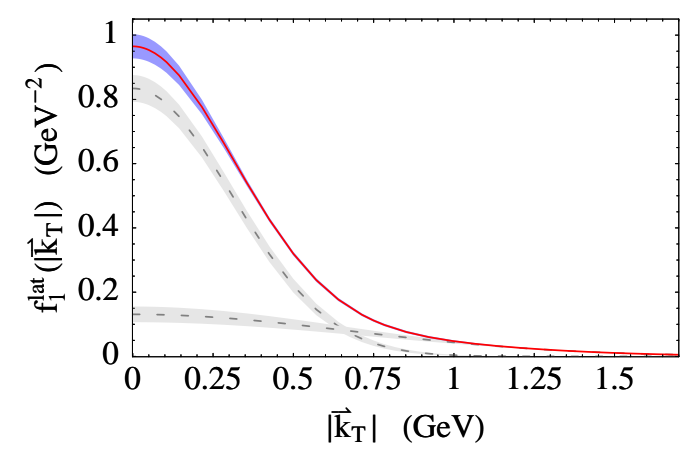

Figure 7: Upper curve: $f_{1, \text { lat }}^{n=1}\left(\vec{k}_{T}\right)$, calculated from a Fourier transform of $R_{\gamma_{4}}(\vec{P}=0,|\vec{\ell}|)$. The upper curve is the sum of the two dashed curves, which show the Fourier transforms of the two Gaussian components in fit function $\mathrm{H}_{2}(|\vec{\ell}|)$. The results are not renormalized. 
where "lat" indicates that the operator has not been renormalized. For the single Gaussian fit (function $H_{1}$ ), we find a root mean square transverse momentum of $\sqrt{\left\langle\vec{k}_{T}^{2}\right\rangle}=2 / \sigma=(563 \pm 12) \mathrm{MeV}$, see table 1 . This is very well compatible with a value of $\approx 500 \mathrm{MeV}$ used in recent phenomenological investigations of HERMES data on SIDIS [10], based on the factorized Ansatz $f_{1}\left(x, \vec{k}_{T}\right)=f_{1}(x) \exp \left[-\vec{k}_{T}^{2} /\left\langle\vec{k}_{T}^{2}\right\rangle\right] /\left[\pi\left\langle\vec{k}_{T}^{2}\right\rangle\right]$. Note however, that such a comparison with phenomenological results has to be taken with due caution, since the effect of renormalization of the non-local operators $\mathscr{O}_{\text {lat }}^{\Gamma}$ could, in principle, affect the $\vec{\ell}$ dependence of $R_{\Gamma}$. The result for the double Gaussian fit (function $\mathrm{H}_{2}$ ) is shown in fig. 7 and table 2. We obtain a root mean square of the transverse momentum of $\sqrt{\left\langle\vec{k}_{T}^{2}\right\rangle}=(702 \pm 12) \mathrm{MeV}$.

\section{Conclusions and outlook}

We have calculated nucleon matrix elements $\left\langle P, S\left|q(0) \Gamma \mathscr{U}_{[0, \ell]} q(\vec{\ell})\right| P, S\right\rangle$ with a finite separation $\ell$ of the quark operators. It turns out that the dependence on $\vec{\ell}$ is approximately Gaussian in the channels we explored. We have used our data to obtain a first, preliminary result on transverse momentum dependent parton distribution functions (TMDPDFs). The root mean square transverse momentum $\sqrt{\left\langle\vec{k}_{T}^{2}\right\rangle}$ of our unrenormalized result for $f_{1}^{n=1}\left(\vec{k}_{T}\right)$ is $(563 \pm 12) \mathrm{MeV}$ for a single Gaussian fit, a value which is compatible with phenomenological results. It will be interesting to study the correlations with respect to the quark separation $\vec{\ell}$ and the nucleon momentum $\vec{P}$. We also plan to investigate whether there is a lattice analogy to link paths extending to infinity and back. This would enable us to calculate the TMDPDFs directly relevant to phenomenology.

\section{Acknowledgments}

Thanks are due to Vladimir Braun for helpful discussions and to the members of the LHPC collaboration for providing propagators and technical expertise. B. M. and $\mathrm{Ph}$. H. acknowledge support by the DFG Emmy Noether-program and A. S. by BMBF. This work was supported in part by funds provided by the U.S. Department of Energy under grant DE-FG02-94ER40818.

\section{References}

[1] J. C. Collins, D. E. Soper and G. Sterman, Phys. Lett. B 134 (1984) 263.

[2] P. J. Mulders and R. D. Tangerman, Nucl. Phys. B 461 (1996) 197 [Erratum-ibid. B 484 (1997) 538].

[3] X. D. Ji, J. P. Ma and F. Yuan, Phys. Rev. D 71 (2005) 034005.

[4] D. Boer, P. J. Mulders and F. Pijlman, Nucl. Phys. B 667 (2003) 201.

[5] J. P. Ralston and D. E. Soper, Nucl. Phys. B 152 (1979) 109.

[6] D. Dolgov et al. [LHPC collaboration], Phys. Rev. D 66 (2002) 034506.

[7] Ph. Hägler et al. [LHPC Collaboration], arXiv:0705.4295 [hep-lat].

[8] K. Orginos and D. Toussaint [MILC collaboration], Phys. Rev. D 59 (1999) 014501.

[9] K. Orginos, D. Toussaint and R. L. Sugar [MILC Collaboration], Phys. Rev. D 60 (1999) 054503.

[10] M. Anselmino, M. Boglione, U. D’Alesio, A. Kotzinian, F. Murgia and A. Prokudin, Phys. Rev. D 71 (2005) 074006. 